FACTA UNIVERSITATIS (NIŠ)

Ser. Math. Inform. Vol. 34, No 2 (2019), 329-339

https://doi.org/10.22190/FUMI1902329I

\title{
OCCURRENCE OF STABLE ROUGH FRACTIONAL INTEGRAL INCLUSION
}

\author{
Rabha W. Ibrahim and Jay M. Jahangiri
}

(C) 2019 by University of Niš, Serbia | Creative Commons Licence: CC BY-NC-ND

Abstract. In the present paper, we generalize the Fredholm type integral operator, by using the fractional rough kernel. We also deal with the Ulam-Hyers stability for rough fractional integral inclusion and utilize the weakly Picard operator method as well as the generalized Covitz-Nadler fixed point theorem.

Keywords. Integral operator; Picard operator method; Fixed point theorem.

\section{Introduction}

The Ulam stability and its generalizations of different functional equations have been studied by various researchers (for recent studies see [2]-[13]). The generalized Ulam-Hyers product-sum stability of the Cauchy type additive functional equation has been investigated by Rassias [14].

Differential equations of arbitrary order were presumed to be models for nonlinear differential equations which played important roles in science, engineering and economics. The studies described computational processes and systems. Consequently, considerable attention has been viewed in the results of fractional differential equations, integral equations, fractional diffeo-integral equations, and fractional partial differential equations of physical phenomena. Most of the studies are concerned with the stability of the solutions [11]-[17].

In this paper, we generalize the Fredholm type integral operator by using the fractional rough kernel and also deal with the Ulam-Hyers stability for rough fractional integral inclusion. We utilize the weakly Picard operator method as well as the generalized Covitz-Nadler fixed point theorem.

Received October 30, 2017; accepted March 14, 2019

2010 Mathematics Subject Classification. Primary 47H10; Secondary 47H11 


\section{Preliminaries}

Let $(E, d)$ be a metric space and let us define the following classes of $E$

$$
\begin{gathered}
\mathcal{P}(E):=\{S \mid S \neq \varnothing\}, \quad \mathcal{P}_{b}(E):=\{S \in \mathcal{P}(E) \mid S \text { is bounded }\}, \\
\mathcal{P}_{c l}(E):=\{S \in \mathcal{P}(E) \mid S \text { is closed }\}, \quad \mathcal{P}_{c p}(E):=\{S \in \mathcal{P}(E) \mid S \text { is compact }\}, \\
\mathcal{P}_{c v}(E):=\{S \in \mathcal{P}(E) \mid S \text { is convex }\} .
\end{gathered}
$$

Let $B\left(e_{0}, r\right):=\left\{e \in E \mid d\left(e_{0}, e\right)<r, r>0\right\}$ be the open ball centered at $e_{0} \in$ $E$. Moreover, denoted by $\bar{B}\left(e_{0}, r\right)$, the closure of $B\left(e_{0}, r\right)$ and $\widetilde{B}\left(e_{0}, r\right):=\{e \in$ $\left.E \mid d\left(e_{0}, e\right) \leq r\right\}$ the closed ball. Define the gap functional in $\mathcal{P}(E)$ by

$$
\mathcal{D}_{d}: \mathcal{P}(E) \times \mathcal{P}(E) \rightarrow \mathbb{R}_{+}, \mathcal{D}_{d}(X, Y)=\inf \{d(x, y) \mid x \in X, y \in Y\}
$$

Also let

$\mathcal{H}_{d}: \mathcal{P}(E) \times \mathcal{P}(E) \rightarrow \mathbb{R}_{+}, \mathcal{H}_{d}(X, Y)=\max \left\{\sup _{x \in X} \mathcal{D}_{d}(x, Y), \sup _{y \in Y} \mathcal{D}_{d}(y, X) \mid x \in X, y \in Y\right\}$,

where $\mathcal{D}_{d}(y, X):=\mathcal{D}_{d}(\{y\}, X)$ and $\mathcal{D}_{d}(x, Y):=\mathcal{D}_{d}(\{x\}, Y)$.

If $G: E \rightarrow \mathcal{P}(E)$ is a multivalued operator, then $g \in E$ is called a fixed point for $G$ iff $g \in G$. The set Fix $(G):=\{g \in E \mid g \in G\}$ is called the fixed point set of $G$. In addition, the set $\operatorname{SFix}(G):=\{g \in E \mid\{g\} \equiv G\}$ is called the strict fixed point set of $G$. For the multi-valued operator $G: E \rightarrow \mathcal{P}(E)$, the graph of $G$ is defined by

$$
\operatorname{Gra}(G):=\{(\phi, \psi) \in E \times S: \psi \in G\} .
$$

Notice that $\gamma: E \rightarrow S$ is a selection for $G: E \rightarrow \mathcal{P}(S)$ if $\gamma(x) \in G(x), x \in E$. We need the following concepts and out comes in the sequel.

Definition 2.1 Let $\varphi: E \rightarrow E$ be an operator and $(E, d)$ be a metric space. Then $\varphi$ is called a weakly Picard operator if the sequence $\left\langle\varphi^{n}\right\rangle_{n \in \mathbb{N}}$ of approximations of $\varphi$ converges and its limit is a fixed point of $\varphi$.

Definition 2.2 Let $(E, \mathfrak{d})$ be a metric space and $\varphi: E \rightarrow E$ be an operator and $\kappa>0$ be a positive constant. Then $\varphi$ is called a $\kappa-$ weakly Picard operator if and only if

$$
\mathfrak{d}\left(\chi, \varphi^{\infty}(\chi)\right) \leq \kappa \mathfrak{d}(\chi, \varphi(\chi)), \quad \forall \chi \in E
$$

where

$$
\varphi^{\infty}: E \rightarrow E, \quad \varphi^{\infty}(\chi):=\lim _{n \rightarrow \infty} \varphi^{n}(\chi)
$$

Definition 2.3 Let $G: E \rightarrow \mathcal{P}_{c l}(E)$ be a multivalued operator on the metric space $(E, \mathfrak{d})$. Then $G$ is called a multivalued weakly Picard operator if for all $\chi \in E$ and $g \in G(\chi)$ there exists a sequence $\left\langle\chi_{n}>_{n \in \mathbb{N}}\right.$ such that 
1. $\chi_{0}=\chi, \chi_{1}=g$;

2. $\chi_{n+1} \in G\left(\chi_{n}\right), \quad n \in \mathbb{N}$;

3. $<\chi_{n}>_{n \in \mathbb{N}} \rightarrow g, g \in \operatorname{Fix}(G)$.

Definition 2.4 Let $\sigma: \mathbb{R}_{+} \rightarrow \mathbb{R}_{+}$be an increasing function which is continuous at 0 and $\sigma(0)=0$ and $(E, \mathfrak{d})$ be a metric space. Then $G: E \rightarrow \mathcal{P}(E)$ is said to be $\sigma-$ weakly Picard operator if it is a multi-valued weakly Picard operator and there exists a selection $\varphi^{\infty}: \operatorname{Gra}(G) \rightarrow \operatorname{Fix}(G)$ such that

$$
\mathfrak{d}\left(\chi, \varphi^{\infty}(\chi, \iota)\right) \leq \sigma(\mathfrak{d}(\chi, \iota)), \quad(\chi, \iota) \in \operatorname{Gra}(G) .
$$

If there exists a constant $\kappa>0$ such that $\sigma(t):=\kappa t$ for each $t \in \mathbb{R}_{+}$, then $G$ is called a multi-valued $\kappa$-weakly Picard operator.

Definition 2.5 $G: E \rightarrow \mathcal{P}_{c l}(E)$ is called a multi-valued $\lambda$-contraction if $\lambda \in[0,1)$ and

$$
\mathcal{H}_{\mathfrak{o}}(G(\chi), G(\iota)) \leq \lambda \mathfrak{d}(\chi, \iota), \quad \forall \chi, \iota \in E,
$$

where $(E, \mathfrak{d})$ is a metric space.

Definition 2.6 Let $G: E \rightarrow \mathcal{P}(E)$ be a multivalued operator, where $(E, \mathfrak{d})$ is a metric space. The fixed point inclusion

$$
u \in G(u), \quad u \in E
$$

is called generalized Ulam-Hyers stable if and only if there exists $\sigma: \mathbb{R}_{+} \rightarrow \mathbb{R}_{+}$ increasing and continuous at 0 and $\sigma(0)=0$ such that for each $\epsilon>0$ and for each solution $\iota^{*} \in E$ of the inequality

$$
\mathcal{D}_{\mathfrak{o}}(\iota, G(\iota)) \leq \epsilon, \quad \iota \in E
$$

there exists a solution $u^{*}$ of $(2.1)$ such that

$$
\mathfrak{d}\left(u^{*}, \iota^{*}\right) \leq \sigma(\epsilon) .
$$

If for $\kappa>0, \sigma(t)=\kappa t, t \in \mathbb{R}_{+}$then the fixed point inclusion (2.1) is said to be $\kappa$-Ulam-Hyers stable.

The following theorem (see Rus [15]) deals with the Ulam-Hyers stability of the fixed point inclusion (2.1).

Theorem 2.1 Let $G: E \rightarrow \mathcal{P}_{c p}(E)$ be a multivalued $\sigma$ - weakly Picard operator and $(E, \mathfrak{d})$ be a metric space. Then the fixed point inclusion $(2.1)$ is generalized Ulam-Hyers stable. 
The following result is a generalization of the Covitz-Nadler fixed point theorem, which can be found in [12] :

Theorem 2.2 Let $(E, d)$ be a complete metric space and $G: E \rightarrow \mathcal{P}_{c l}(E)$ be a multivalued $\lambda$ - contraction operator $\mathcal{H}_{d}\left(G\left(u_{1}\right), G\left(u_{2}\right)\right) \leq \lambda d\left(u_{1}, u_{2}\right), \quad \forall u_{1}, u_{2} \in E$. Then $\operatorname{Fix}(G)$ is nonempty and for $u_{0} \in E$ there exists a sequence of approximations of $G$ starting from $u_{0}$ which converges to a fixed point of $G$.

Next Ulam-Hyers stability result, which is very useful for applications, was introduced in [10].

Theorem 2.3 Let $(E, \mathfrak{d})$ be a complete metric space and $G: E \rightarrow \mathcal{P}_{c l}(E)$ be a multi-valued $\lambda$ - contraction operator. Then

1. $G$ is a multi-valued weakly Picard operator;

2. If $\psi(a t) \leq a \psi(t)$ for every $t \in \mathbb{R}_{+}, a>1$ and the series $\tau(t):=\sum_{n=1}^{\infty} \varphi^{n}(t)$ converges to the point $t=0$, then $G$ is a $\sigma$-multivalued weakly Picard operator with $\sigma(t):=t+\tau(t)$ and $t \in \mathbb{R}_{+}$;

3. Let $Q: E \rightarrow \mathcal{P}_{c l}(E)$ be a multi-valued $\lambda$-contraction and $b>0$ such that $\mathcal{H}(Q(\chi), G(\chi)) \leq b, \chi \in E$. Suppose that $\lambda(a t) \leq a \lambda(t), t \in \mathbb{R}_{+}, a>1$ and the series $\tau(t)$ converges uniformly to the point $t=0$. Then $\mathcal{H}(\operatorname{Fix}(Q), \operatorname{Fix}(G)) \leq$ $\sigma(b)$.

\section{Ulam Stability}

In this section, we further investigate the Ulam stability by utilizing the above mentioned concepts and results. Recently, Ibrahim and Jalab [8] established the existence of solutions for integral inclusion of fractional order in the sense of RiemannLiouville integral operator. We consider the following fractional integral inclusion:

$$
u(t) \in \int_{a}^{b} G(t, \varsigma, u(\varsigma)) \frac{\Omega(\varsigma)}{\varsigma^{n-\alpha}} d \varsigma+g(t),
$$

where $u \in \mathbb{R}^{n}, \Omega$ belongs to the unit sphere of $\mathbb{R}^{n}, 0<\alpha<n$ and $t, \varsigma \in J:=$ $[a, b], a, b>0$. When $\Omega \equiv 1$ and $\alpha \rightarrow n$, inclusion(3.1) reduces to the Fredholm type integral inclusion. We have the following result:

Theorem 3.1 Let $G: J \times J \times \mathbb{R}^{n} \rightarrow \mathcal{P}_{c l, c v}, \Omega: J \rightarrow \mathbb{R}^{n}$ and $g: J \rightarrow \mathbb{R}^{n}$ such that

1. There exists an integrable function $\imath: J \rightarrow \mathbb{R}_{+}^{n}$ such that $G(t, \varsigma, u) \subset \imath(\varsigma) \times$ $B(0,1), t, \varsigma \in J, u \in \mathbb{R}^{n}=E ;$ 
2. $G(., ., u): J \times J \times \mathbb{R}^{n} \rightarrow \mathcal{P}_{c l, c v}$ is jointly measurable for all $u \in \mathbb{R}^{n}$;

3. $G(., \varsigma, u): J \times J \times \mathbb{R}^{n} \rightarrow \mathcal{P}_{c l, c v}$ is lower semi-continuous for all $(\varsigma, u) \in\left(J, \mathbb{R}^{n}\right)$;

4. There exist a continuous function $\rho: J \times J \rightarrow \mathbb{R}_{+}$with $\sup _{t \in J} \int_{a}^{b} \rho(t, \varsigma) d \varsigma \leq 1$ and a positive function $\theta: \mathbb{R}_{+} \rightarrow \mathbb{R}_{+}$satisfying

$$
\mathcal{H}(G(t, \varsigma, u), G(t, \varsigma, v)) \leq \rho(t, \varsigma) \cdot \theta(|u-v|)
$$

5. $\Omega$ and $g$ are continuous;

6. $\|\Omega\|:=\sup _{s \in J}|\Omega(s)|$; with $\frac{\|\Omega\|}{a^{n-\alpha}}<1$.

Then the following conclusions hold

1. Inclusion (3.1) has at least one solution $u^{*} \in C\left(J, \mathbb{R}^{n}\right)$;

2. If the series $\sum_{n=1}^{\infty} \theta^{n}$ converges uniformly to $t=0$, where $\theta(q t) \leq q \theta(t)$ for every $t \in \mathbb{R}_{+}, q>1$, then the fractional integral inclusion (3.1) is generalized Ulam-Hyers with function $\sigma$, where $\sigma(t)=t+\varsigma(t)$ for each $t \in \mathbb{R}_{+}$and $\varsigma(t):=\sum_{n=1}^{\infty} \theta^{n}$. Equivalently, for each $\epsilon>0$ and $v \in C\left(J, \mathbb{R}^{n}\right)$ there exists $u \in C\left(J, \mathbb{R}^{n}\right)$ such that

$$
\begin{gathered}
u(t) \in \int_{a}^{b} G(t, \varsigma, v(\varsigma)) \frac{\Omega(\varsigma)}{|\varsigma|^{n-\alpha}} d \varsigma+g(t), \\
|u(t)-v(t)| \leq \epsilon, \quad t \in J
\end{gathered}
$$

and

$$
\left|v(t)-u^{*}(t)\right| \leq \sigma(\epsilon), \quad t \in J
$$

Proof. Define the multivalued operator $M: C\left(J, \mathbb{R}^{n}\right) \rightarrow \mathcal{P}\left(C\left(J, \mathbb{R}^{n}\right)\right)$ by

$$
M(u):=\left\{v \in C\left(J, \mathbb{R}^{n}\right) \mid v(t) \in \int_{a}^{b} G(t, \varsigma, u(\varsigma)) \frac{\Omega(\varsigma)}{\varsigma^{n-\alpha}} d \varsigma+g(t)\right\} .
$$

Then (3.1) is equivalent to the fixed point inclusion

$$
u \in M(u), \quad u \in C\left(J, \mathbb{R}^{n}\right) .
$$

The rest of the proof will be given in three steps.

Step 1. $M(u) \in \mathcal{P}_{c p}\left(C\left(J, \mathbb{R}^{n}\right)\right)$. 
By the continuity of $\Omega$ and $g$, we obtain $\gamma(t, \varsigma) \in G(t, \varsigma, u), t, \varsigma \in J$ such that

$$
v(t):=\int_{a}^{b} \gamma(t, \varsigma) \frac{\Omega(\varsigma)}{\varsigma^{n-\alpha}} d \varsigma \in M(u) .
$$

In view of [1], Theorem 8.6.3, together with the hypotheses 1 and 2 we conclude that $M(u)$ is a compact set for all $u \in C\left(J, \mathbb{R}^{n}\right)$.

Step 2. $\mathcal{H}\left(M\left(u_{1}\right), M\left(u_{2}\right)\right) \leq \theta\left(\left\|u_{1}-u_{2}\right\|\right), \quad u_{1}, u_{2} \in C\left(J, \mathbb{R}^{n}\right)$.

For $u_{1}, u_{2} \in C\left(J, \mathbb{R}^{n}\right)$, we let $v_{1} \in M\left(u_{1}\right)$. Thus

$$
v_{1}(t) \in \int_{a}^{b} G\left(t, \varsigma, u_{1}(\varsigma)\right) \frac{\Omega(\varsigma)}{\varsigma^{n-\alpha}} d \varsigma+g(t) .
$$

Therefore there is an integrable function $\gamma_{1}$ such that

$$
v_{1}(t)=\int_{a}^{b} \gamma_{1}(t, \varsigma) \frac{\Omega(\varsigma)}{\varsigma^{n-\alpha}} d \varsigma+g(t) .
$$

In virtue of the assumption 4 , we conclude that

$$
H\left(G\left(t, \varsigma, u_{1}\right), G\left(t, \varsigma, u_{2}\right)\right)<\rho(t, \varsigma) \theta\left(\left|u_{1}(\varsigma)-u_{2}(\varsigma)\right|\right) \leq \rho(t, \varsigma) \theta\left(\left\|u_{1}-u_{2}\right\|\right) .
$$

So, there exists $w \in G\left(t, \varsigma, u_{2}(\varsigma)\right)$ such that

$$
\left|\gamma_{1}(t, \varsigma)-w\right| \leq \rho(t, \varsigma) \theta\left(\left\|u_{1}-u_{2}\right\|\right), \quad t, \varsigma \in J .
$$

Define a set $\Gamma(t, \varsigma)$ by

$$
\Gamma(t, \varsigma):=\left\{w \| \gamma_{1}(t, \varsigma)-w \mid \leq \rho(t, \varsigma) \theta\left(\left\|u_{1}-u_{2}\right\|\right)\right\}
$$

and a multivalued operator by

$$
\Theta(t, \varsigma):=\Gamma(t, \varsigma) \bigcap G\left(t, \varsigma, u_{2}(\varsigma)\right)
$$

Thus according to the assumptions 2 and $3, \Theta$ is jointly measurable and lower semicontinuous in $t$. Consequently, there exists $\gamma_{2}(t, \varsigma)$ a selection for $\Theta$, jointly measurable, integrable in $\varsigma$ and lower semi-continuous in $t$. Hence $\gamma_{2}(t, \varsigma) \in G\left(t, \varsigma, u_{2}\right)$ and

$$
\left|\gamma_{1}(t, \varsigma)-\gamma_{2}(t, \varsigma)\right| \leq \rho(t, \varsigma) \theta\left(\left\|u_{1}-u_{2}\right\|\right), \quad t, \varsigma \in J
$$

Consider

$$
v_{2}(t) \in \int_{a}^{b} G\left(t, \varsigma, u_{2}(\varsigma)\right) \frac{\Omega(\varsigma)}{\varsigma^{n-\alpha}} d \varsigma+g(t)
$$

with

$$
v_{2}(t)=\int_{a}^{b} \gamma_{2}(t, \varsigma) \frac{\Omega(\varsigma)}{\varsigma^{n-\alpha}} d \varsigma+g(t) .
$$


Then, by utilizing assumption 6 , we get

$$
\left|v_{1}(t)-v_{2}(t)\right| \leq \frac{\|\Omega\|}{a^{n-\alpha}} \int_{a}^{b} \rho(t, \varsigma) \theta\left(\left\|u_{1}-u_{2}\right\|\right) d \varsigma \leq \theta\left(\left\|u_{1}-u_{2}\right\|\right) .
$$

Similar arguments can be used for $u_{1}$ and $u_{2}$. Hence, in view of Theorem 2.2, inclusion (3.1) has a solution.

Step 3. Generalized Ulam-Hyers stable.

Now, our aim is to show that the fixed point inclusion (3.3) is generalized UlamHyers stable. Let $\epsilon>0$ and $\mu \in C\left(J, \mathbb{R}^{n}\right)$ for which there exists $u \in C\left(J, \mathbb{R}^{n}\right)$ such that

and

$$
u(t) \in \int_{a}^{b} G(t, \varsigma, \mu(\varsigma)) \frac{\Omega(\varsigma)}{|\varsigma|^{n-\alpha}} d \varsigma+g(t), \quad t \in J
$$

This implies that

$$
\|u-\mu\| \leq \epsilon
$$

$\mathcal{D}_{\|\cdot\|}(\mu, M(\mu)) \leq \epsilon$.

Since $M$ is a multivalued $\theta$-contraction and applying Theorem 2.3, we have that $M$ is a multivalued $\sigma$-weakly Picard operator. Then according to Theorem 2.1, we conclude that the fixed point problem (3.3) is generalized Ulam-Hyers stable. Therefore, the fractional integral inclusion (3.1) is generalized Ulam-Hyers stable. For the last assertion, we utilize Theorem 2.3, which completes the proof.

Next, we consider the following fractional integral inclusion

$$
u(t) \in \int_{a}^{t} K(t, \varsigma, u(\varsigma)) \frac{\Omega(\varsigma)}{\varsigma^{n-\alpha}} d \varsigma+h(t)
$$

where $u \in \mathbb{R}^{n}, \Omega$ belongs to the unit sphere of $\mathbb{R}^{n}, 0<\alpha<n$ and $t, \varsigma \in J=$ $[a, b], a, b>0$. When $\Omega \equiv 1$ and $\alpha \rightarrow n$, inclusion (3.4) reduces to the Volterra type integral inclusion. In the same manner as of Theorem 3.1, we have the following

Theorem 3.2 Let $K: J \times J \times \mathbb{R}^{n} \rightarrow \mathcal{P}_{c l, c v}, \Omega: J \rightarrow \mathbb{R}^{n}$ and $h: J \rightarrow \mathbb{R}^{n}$ such that

1. There exists an integrable function $\imath: J \rightarrow \mathbb{R}_{+}^{n}$ such that $K(t, \varsigma, u) \subset \imath(\varsigma) \times$ $B(0,1), t, \varsigma \in J, u \in \mathbb{R}^{n}=E ;$

2. $K(., ., u): J \times J \times \mathbb{R}^{n} \rightarrow \mathcal{P}_{c l, c v}$ is jointly measurable for all $u \in \mathbb{R}^{n}$;

3. $K(., \varsigma, u): J \times J \times \mathbb{R}^{n} \rightarrow \mathcal{P}_{c l, c v}$ is lower semi-continuous for all $(\varsigma, u) \in\left(J, \mathbb{R}^{n}\right)$;

4. There exist a continuous function $\rho: J \times J \rightarrow \mathbb{R}_{+}$with $\sup _{t \in J} \int_{a}^{t} \rho(t, \varsigma) d \varsigma \leq 1$ and a positive function $\theta: \mathbb{R}_{+} \rightarrow \mathbb{R}_{+}$satisfying

$$
\mathcal{H}(K(t, \varsigma, u), K(t, \varsigma, v)) \leq \rho(t, \varsigma) \cdot \theta(|u-v|)
$$


5. $\Omega$ and $h$ are continuous;

6. $\|\Omega\|:=\sup _{s \in J}|\Omega(s)| ;$ with $\frac{\|\Omega\|}{a^{n-\alpha}}<1$.

Then the following conclusions hold:

1. Inclusion (3.4) has at least one solution $u^{*} \in C\left(J, \mathbb{R}^{n}\right)$;

2. If the series $\sum_{n=1}^{\infty} \theta^{n}$ converges uniformly to $t=0$, where $\theta(q t) \leq q \theta(t)$ for every $t \in \mathbb{R}_{+}, q>1$, then the fractional integral inclusion (3.4) is generalized Ulam-Hyers with function $\sigma$, where $\sigma(t)=t+\varsigma(t)$ for each $t \in \mathbb{R}_{+}$and $\varsigma(t):=\sum_{n=1}^{\infty} \theta^{n}$. Equivalently, for each $\epsilon>0$ and $v \in C\left(J, \mathbb{R}^{n}\right)$ there exists $u \in C\left(J, \mathbb{R}^{n}\right)$ such that

$$
\begin{aligned}
u(t) \in & \int_{a}^{b} K(t, \varsigma, v(\varsigma)) \frac{\Omega(\varsigma)}{\varsigma^{n-\alpha}} d \varsigma+h(t), \\
& |u(t)-v(t)| \leq \epsilon, \quad t \in J
\end{aligned}
$$

and

$$
\left|v(t)-u^{*}(t)\right| \leq \sigma(\epsilon), \quad t \in J
$$

Proof. Define the multivalued operator $V: C\left(J, \mathbb{R}^{n}\right) \rightarrow \mathcal{P}\left(C\left(J, \mathbb{R}^{n}\right)\right)$ by

$$
V(u):=\left\{v \in C\left(J, \mathbb{R}^{n}\right) \mid v(t) \in \int_{a}^{t} K(t, \varsigma, u(\varsigma)) \frac{\Omega(\varsigma)}{\varsigma^{n-\alpha}} d \varsigma+h(t)\right\} .
$$

Then (3.4) is equivalent to the fixed point inclusion

$$
u \in V(u), \quad u \in C\left(J, \mathbb{R}^{n}\right) .
$$

In a similar method as of Theorem 3.1 , we may have $V(u) \in \mathcal{P}_{c p}\left(C\left(J, \mathbb{R}^{n}\right)\right)$. Now we proceed to show that $V$ is $\theta$-contraction mapping on $C\left(J, \mathbb{R}^{n}\right)$.

For $u_{1}, u_{2} \in C\left(J, \mathbb{R}^{n}\right)$, we let $v_{1} \in V\left(u_{1}\right)$. Thus

$$
v_{1}(t) \in \int_{a}^{t} K\left(t, \varsigma, u_{1}(\varsigma)\right) \frac{\Omega(\varsigma)}{\varsigma^{n-\alpha}} d \varsigma+h(t) .
$$

Therefore, there is an integrable function $\gamma_{1}$ such that

$$
v_{1}(t)=\int_{a}^{t} \gamma_{1}(t, \varsigma) \frac{\Omega(\varsigma)}{\varsigma^{n-\alpha}} d \varsigma+h(t) .
$$

In view of the assumption 4 , we conclude that

$$
H\left(K\left(t, \varsigma, u_{1}\right), K\left(t, \varsigma, u_{2}\right)\right)<\rho(t, \varsigma) \theta\left(\left|u_{1}(\varsigma)-u_{2}(\varsigma)\right|\right) \leq \rho(t, \varsigma) \theta\left(\left\|u_{1}-u_{2}\right\|\right)
$$


Thus, there exists $w \in K\left(t, \varsigma, u_{2}(\varsigma)\right)$ such that

$$
\left|\gamma_{1}(t, \varsigma)-w\right| \leq \rho(t, \varsigma) \theta\left(\left\|u_{1}-u_{2}\right\|\right), \quad t, \varsigma \in J .
$$

Define a set $\Lambda(t, \varsigma)$ by

$$
\Lambda(t, \varsigma):=\left\{w:\left|\gamma_{1}(t, \varsigma)-w\right| \leq \rho(t, \varsigma) \theta\left(\left\|u_{1}-u_{2}\right\|\right)\right\}
$$

and a multivalued operator by

$$
\Psi(t, \varsigma):=\Lambda(t, \varsigma) \bigcap K\left(t, \varsigma, u_{2}(\varsigma)\right)
$$

Therefore, according to the assumptions 2 and $3, \Psi$ is jointly measurable and lower semi-continuous in $t$. Consequently, there exists $\gamma_{2}(t, \varsigma)$ a selection for $\Psi$, jointly measurable, integrable in $\varsigma$ and lower semi-continuous in $t$. Hence, $\gamma_{2}(t, \varsigma) \in$ $K\left(t, \varsigma, u_{2}\right)$ and

$$
\left|\gamma_{1}(t, \varsigma)-\gamma_{2}(t, \varsigma)\right| \leq \rho(t, \varsigma) \theta\left(\left\|u_{1}-u_{2}\right\|\right), \quad t, \varsigma \in J
$$

Consider

$$
v_{2}(t) \in \int_{a}^{t} K\left(t, \varsigma, u_{2}(\varsigma)\right) \frac{\Omega(\varsigma)}{\varsigma^{n-\alpha}} d \varsigma+h(t)
$$

with

$$
v_{2}(t)=\int_{a}^{t} \gamma_{2}(t, \varsigma) \frac{\Omega(\varsigma)}{\varsigma^{n-\alpha}} d \varsigma+h(t) .
$$

Define a norm by

$$
\|u\|_{\mathcal{B}}:=\sup _{t \in J}\left(\left|u(t) e^{-q(t)}\right|\right)
$$

where $q(t):=\int_{a}^{t} \rho(\varsigma) d \varsigma$. Then by using the assumption 6 , we get

$$
\begin{aligned}
\left|v_{1}(t)-v_{2}(t)\right| & \left.\leq \frac{\|\Omega\|}{a^{n-\alpha}} \int_{a}^{t}\left|\gamma_{1}(t, \varsigma)-\gamma_{2}(t, \varsigma)\right|\right) d \varsigma \\
& \leq \frac{\|\Omega\|}{a^{n-\alpha}} \int_{a}^{t} \rho(t, \varsigma) \theta\left(\left|u_{1}(\varsigma)-u_{2}(\varsigma)\right|\right) d \varsigma \\
& \leq \frac{\|\Omega\|}{a^{n-\alpha}} \int_{a}^{t} \rho(t, \varsigma) \theta\left(e^{q(\varsigma)}\left|u_{1}(\varsigma)-u_{2}(\varsigma)\right| e^{-q(\varsigma)}\right) d \varsigma \\
& \leq \frac{\|\Omega\|}{a^{n-\alpha}} \int_{a}^{t} \rho(t, \varsigma) e^{q(\varsigma)} \theta\left(\left\|u_{1}-u_{2}\right\|_{\mathcal{B}}\right) \\
& \leq \frac{\|\Omega\|}{a^{n-\alpha}} \theta\left(\left\|u_{1}-u_{2}\right\|_{\mathcal{B}}\right)\left(e^{q(t)}-e^{q(a)}\right) \\
& \leq \theta\left(\left\|u_{1}-u_{2}\right\|_{\mathcal{B}}\right) e^{q(t)} .
\end{aligned}
$$

Thus, we have

$$
\left\|v_{1}-v_{2}\right\|_{\mathcal{B}} \leq \theta\left(\left\|u_{1}-u_{2}\right\|_{\mathcal{B}}\right)
$$


Hence, in view of Theorem 2.2, inclusion (3.4) has a solution.

It suffices to prove that the fixed point inclusion (3.6) is generalized Ulam-Hyers stable. Let $\epsilon>0$ and $\nu \in C\left(J, \mathbb{R}^{n}\right)$ for which there exists $u \in C\left(J, \mathbb{R}^{n}\right)$ such that

$$
u(t) \in \int_{a}^{t} K(t, \varsigma, \nu(\varsigma)) \frac{\Omega(\varsigma)}{|\varsigma|^{n-\alpha}} d \varsigma+h(t), \quad t \in J
$$

and

$$
\left\|v_{1}-v_{2}\right\|_{\mathcal{B}} \leq\|u-\nu\| \leq \epsilon
$$

This implies that

$$
\mathcal{D}_{\|\cdot\|_{\mathcal{B}}}(\nu, V(\nu)) \leq \epsilon
$$

Since $V$ is a multi-valued $\theta$-contraction with respect to the norm $\|\cdot\|_{\mathcal{B}}$, then $V$ is a multi-valued weakly operator. Using Theorem 2.3 , we have that $V$ is a multi-valued $\sigma$-weakly Picard operator. Then according to Theorem 2.1, we conclude that the fixed point problem (3.6) is generalized Ulam-Hyers stable. This implies that there exists a solution $u^{*}$ of inclusion (3.4) such that

$$
\left\|\nu-u^{*}\right\|_{\mathcal{B}} \leq \sigma(\epsilon), \quad \epsilon>0 .
$$

Thus, we have

$$
\left|\nu-u^{*}\right| \leq \sigma\left(e^{t q(b)} \epsilon\right), \quad t \in J=[a, b] .
$$

Theorem 2.3 yields the last conclusion and this completes the proof.

Acknowledgments: The authors would like to express their thanks to the reviewers for their important and useful comments to improve the paper.

\section{R E F E R E N C E S}

1. J.P. Aubin, H. Frankowska, Set-Valued Analysis, Birkhäuser, Basel, 1990.

2. P. Găvruță, On a problem of G. Isac and Th. Rassias concerning the stability of mapping, J. Math. Anal. Appl., 261(2001), 543-553.

3. D. H. Hyers and T. M. Rassias, Approximate homomorphisms, Aequationes Mathematicae, 44(1992) 125-153.

4. D. H. Hyers, G. Isac, Th.M. Rassias, Stability of Functional Equations in Several Variables, Birkhäuser, Basel, 1998.

5. R. W. Ibrahim, Ulam stability for fractional differential equation in complex domain, Abstract and Applied Analysis, Volume 2012, Article ID 649517, 8 pages doi:10.1155/2012/649517.

6. R. W. Ibrahim, Generalized Ulam-Hyers stability for fractional differential equations, Int. J. Math., 23( 5) (2012) 1-9.

7. R. W. Ibrahim, Ulam-Hyers stability for Cauchy fractional differential equation in the unit disk, Abstract and Applied Analysis, vol. 2012, Article ID 613270, 10 pages doi:10.1155/2012/613270. 
8. R. W. Ibrahim, H. A. Jalab, Existence of the solution of fractional integral inclusion with time delay, Miskolc Mathematical Notes, 11 (2010) 139-150.

9. S.-M. Jung, Hyers-Ulam-Rassias Stability of Functional Equations in Mathematical Analysis, Hadronic Press, Palm Harbor, 2001.

10. V.L. Lazăr, Fixed point theory for multivalued $\varphi$-contractions, Fixed Point Theory and Applications, vol. 2011:50, (2011) 1-12

11. Y. Li, Y. Chen, I. Podlubny, Stability of fractional-order nonlinear dynamic systems: Lyapunov direct method and generalized Mittag-Leffler stability. Comput Math Appl 59 (2010) 1810-1821.

12. S. B. Nadler, Multivalued contraction mappings, Pacific J. Math., 30(1969) 475488.

13. V. Radu, The fixed point alternative and the stability of functional equations, Fixed Point Theory, 4(2003) 91-96.

14. M. J. Rassias, Generalised Hyers-Ulam product-sum stability of a Cauchy type additive functional equation, European Journal of Pure and Applied Mathematics, 4 (2011) 50-58.

15. I.A. Rus, Remarks on Ulam stability of the operatorial equations, Fixed Point Theory, 10(2009) 305-320.

16. J. Wang, L. Lv, Y. Zhou, Ulam stability and data dependence for fractional differential equations with Caputo derivative. Electron J Qualit Th Diff Equat 63( 2011) 1-10.

17. Q. Yu, F. Liu, I. Turner, K. Burrage, Stability and convergence of an implicit numerical method for the space and time fractional Bloch-Torrey equation. Phil. Trans. R. Soc. A, 371 (2013).

Rabha W. Ibrahim

IEEE: 94086547

rabhaibrahim@yahoo.com

Jay M. Jahangiri

Department of Mathematical Sciences

Kent State University, Kent, Ohio, U.S.A.

jjahangi@kent.edu 